Check for updates

Cite this: RSC Adv., 2018, 8, 9287

Received 3rd November 2017

Accepted 5th February 2018

DOI: $10.1039 / c 7 r a 12087 g$

rsc.li/rsc-advances

\title{
The preparation of a three dimensional terbium doped reduced graphene oxide aerogel with photoluminescence and paramagnetic properties $\uparrow$
}

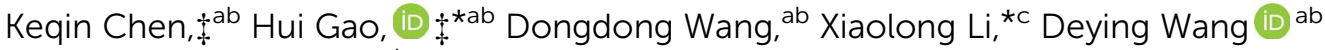 \\ and Waheed Ullah Khan ${ }^{\text {ab }}$
}

\begin{abstract}
A 3D reduced graphene oxide $(\mathrm{rGO})$ material with good photoluminescence $(\mathrm{PL})$ and magnetism properties was self-assembled using the hydrothermal method. The resultant material possessed a flower-like structure, which leads to a high surface area. To dehydrate the hydrogel for conservation, the sublimation process we use can effectively maintain the porosity as it uses the freeze drying method. Then, rGO was endowed with paramagnetism and green photoluminescence properties via the introduction of $\mathrm{Tb}$ ions, which also reinforces the significance of the building block. Finally, the coordination between $\mathrm{Tb}$ ions and the carbonyl was proven with photoluminescence excitation (PLE) spectra and the UV-vis absorption spectra, which are likely to offer an effective way to study the carbonyl compound.
\end{abstract}

\section{Introduction}

Graphene has attracted great interest in research fields, due to its excellent electrical properties and high specific surface area. ${ }^{1,2}$ However, finely-sized graphene causes environmental pollution and suffers from poor integral mechanical properties while aggregated graphene monoliths lose the large surface area. ${ }^{3,4}$ Thus, it is necessary to integrate graphene sheets into 3D porous structures..$^{5-7}$ So far, some work has made considerable progress in the synthesis of novel configurations, such as millispheres and plant stem structures. ${ }^{3,5}$ Another research hot topic focuses on the multi-functionalization of graphene, leading to the tremendous development of graphene and its derivatives, such as in electrodes, catalysis carriers, sensors and chemical filters. $^{8-11}$ Graphene is also considered a promising spintronic device owing to its room-temperature spin transport and long spin diffusion lengths. ${ }^{12}$ However, pristine graphene is usually intrinsically diamagnetic. Introducing some foreign atoms or defects in the delocalized $\pi$ bonding network of

${ }^{a}$ School of Physical Science and Technology, Key Laboratory for Magnetism and Magnetic Materials of Ministry of Education, Lanzhou University, Lanzhou 730000 , P. R. China. E-mail: hope@lzu.edu.cn; Fax: +86-931-8913554; Tel: +86-18993181143 ${ }^{b}$ National \& Local Joint Engineering Laboratory for Optical Conversion Materials and Technology, Key Laboratory of Special Function Materials and Structure Design, Ministry of Education, P. R. China

'Shanghai Synchrotron Radiation Facility, Shanghai Institute of Applied Physics, Chinese Academy of Sciences, Shanghai 201204, P. R. China. E-mail: lixiaolong@ sinap.ac.cn

$\dagger$ Electronic supplementary information (ESI) available. See DOI: $10.1039 / \mathrm{c} 7 \mathrm{ra} 12087 \mathrm{~g}$

$\$$ These authors contributed equally to the article. graphene is a widely utilized method in its practical application or in theoretical calculations to tailor its magnetism. ${ }^{13,14}$ And it is imperative to study the exact site where foreign ions are located, as not knowing this prevents us from understanding the system, and even the ion exchange process in lithium ion batteries and filter systems.

Our group has focused on rare earth ion doped materials, which possess good photoluminescence properties, high fluorescence quantum efficiencies, and low toxicities. The compounds ( $\mathrm{rGO} /$ europium and $\mathrm{rGO} / \mathrm{Tb}$ ) had excellent luminescence properties and quenching abilities with rhodamine-B in our previous work. ${ }^{15,16}$ Meanwhile, terbium (Tb) plays a crucial role among the lanthanum elements, owing to its characteristic green emission and paramagnetism at room temperature. Thus, $\mathrm{Tb}$ can be exploited to tune magnetism and determine binding sites by measuring its optical behavior.

As a rich porous texture is prepared using the hydrothermal method, the dry method is a crucial link in the production chain, directly related to the mechanical strength and the surface area. ${ }^{5}$ So it is a challenge to clarify the link between the dry method and the pore properties. Besides, photoluminescence (PL) has become a technique used to study acceptor-donor recombination and the defects associated with trap levels. ${ }^{10}$ There are few studies on the binding sites of dopants in graphene using the PL method.

Herein, we report a 3D multifunctional cylinder, reduced graphene oxide doped with $\mathrm{Tb}$ ions, readily obtained by the hydrothermal method. Two main factors, the amount of water and use of the dry method, are studied in the 3D building process. The photoluminescence behavior of $\mathrm{Tb}$ ions and graphene indicates an energy-transfer process between them as do 
the binding sites of the Tb ions. It is noted that the introduction of $\mathrm{Tb}$ endows graphene with paramagnetism. In general, the photoluminescence and paramagnetic properties of the resultant composite are based on 3D modeling. Hence, this 3D structure and multifunctional material can potentially be applied in 3D devices and models.

\section{Experimental}

\subsection{Materials}

$\mathrm{Tb}_{4} \mathrm{O}_{7}$ (99.9\%) was purchased from Shanghai Chemical Company. Potassium permanganate, hydrogen peroxide, potassium peroxodisulfate, phosphorus pentoxide, graphite powder, sodium nitrate, concentrated sulfuric acid and hydrochloric acid were all analytically pure. GO was synthesized using the modified Hummers method. $\mathrm{Tb}_{4} \mathrm{O}_{7}$ was dissolved in hydrochloric acid, and dried at $333 \mathrm{~K}$ for $24 \mathrm{~h}$ to obtain rGO-0, namely $\mathrm{TbCl}_{3}$.

\subsection{Preparation of a series of rGO aerogels}

A certain amount of $\mathrm{TbCl}_{3}$ aqueous solutions were dispersed into GO. Then, the dispersions were transferred into a Teflon tube and heated at $453 \mathrm{~K}$ for $8 \mathrm{~h}$ to obtain hydrogels. The different cylinder-like sponges were obtained after freeze-drying treatment. rGO-1 is made of $15 \mathrm{ml}$ of purified water and $30 \mathrm{ml}$ of GO (2 mg ml $\mathrm{m}^{-1}$ ); rGO-2 is made of $15 \mathrm{ml}$ of $\mathrm{TbCl}_{3}$ aqueous solution $\left(0.3 \mathrm{mg} \mathrm{ml}^{-1}\right)$ and $30 \mathrm{ml}$ of GO $\left(2 \mathrm{mg} \mathrm{ml}^{-1}\right)$; rGO-3 is made of $2.5 \mathrm{ml}$ of purified water and $20 \mathrm{ml}$ of $\mathrm{GO}\left(3 \mathrm{mg} \mathrm{ml}^{-1}\right)$; rGO-4 is made of $2.5 \mathrm{ml}$ of $\mathrm{TbCl}_{3}$ aqueous solution $\left(2 \mathrm{mg} \mathrm{ml}^{-1}\right)$ and $20 \mathrm{ml}$ of GO $\left(3 \mathrm{mg} \mathrm{ml}^{-1}\right)$; rGO-5 is made of $2.5 \mathrm{ml}$ of $\mathrm{TbCl}_{3}$ aqueous solution $\left(2 \mathrm{mg} \mathrm{ml}^{-1}\right)$ and $20 \mathrm{ml}$ of $\mathrm{GO}\left(3 \mathrm{mg} \mathrm{ml} \mathrm{mo}^{-1}\right)$ and exposed to air for 1 day before freeze-drying treatment.
Here, the liquid volume $(22.5 \mathrm{ml}$ and $45 \mathrm{ml})$ is a factor affecting the geometrical parameters and magnetism properties of rGO, as shown in Fig. 1.

\subsection{Characterization}

The sample's morphology was examined using scanning electron microscopy (SEM, Hitachi, S-4800), powder X-ray diffraction (XRD) (Rigaku, Japan), X-ray photoelectron spectroscopy (XPS, PHI-5702, Physical Electronics), Raman spectroscopy (Renishaw InVia Raman microscope), Fourier transform infrared spectroscopy (Nicolet NEXUS 670 FTIR) and transmission electron microscopy (TEM, Tecnai G2, FEI Company). The PL spectra were obtained with an FLS-920T fluorescence spectrophotometer. The magnetization was characterized using a vibrating sample magnetometer (VSM). The thickness of rGO2 was measured using atomic force microscopy (AFM) (Digital instruments Nanoscope III A).

\section{Results and discussion}

XPS measurement is a practical analytical approach to investigate the chemical composition and surface electronic state. The XPS spectrum shows the presence of carbon, oxygen and terbium atoms, as shown in Fig. S1a. $\uparrow$ The $\mathrm{C} 1 \mathrm{~s}$ peak is divided into three Gaussian peaks centered at 284.5, 285.5 and $287.8 \mathrm{eV}$, suggesting different energies of carbon. The binding energies $284.5 \mathrm{eV}, 285.5 \mathrm{eV}$ and $287.7 \mathrm{eV}$ match the present $\mathrm{sp}^{2}$ hybridized $\mathrm{C}$ atoms $(\mathrm{C}=\mathrm{C})$, the bond between $\mathrm{C}$ and $\mathrm{O}(\mathrm{C}-\mathrm{O})$, and the carbonyl $(\mathrm{C}=\mathrm{O})$, respectively. The $\mathrm{O}$ 1s core level spectrum shown in Fig. S1b † contains two peaks, centered at $530.8 \mathrm{eV}$ and $533.1 \mathrm{eV}$. It has been reported that the binding energy of the $\mathrm{O}-\mathrm{H}$ bond in the hydroxyl group $(\mathrm{O}-\mathrm{H})$ should be $529.0-$ $531.1 \mathrm{eV}$, while it is approximately $533.0 \mathrm{eV}$ for $\mathrm{C}-\mathrm{OH}, \mathrm{C}=\mathrm{O}$, and $\mathrm{O}=\mathrm{C}-\mathrm{OH}$. The binding energy related to $\mathrm{Tb}$ is clarified in

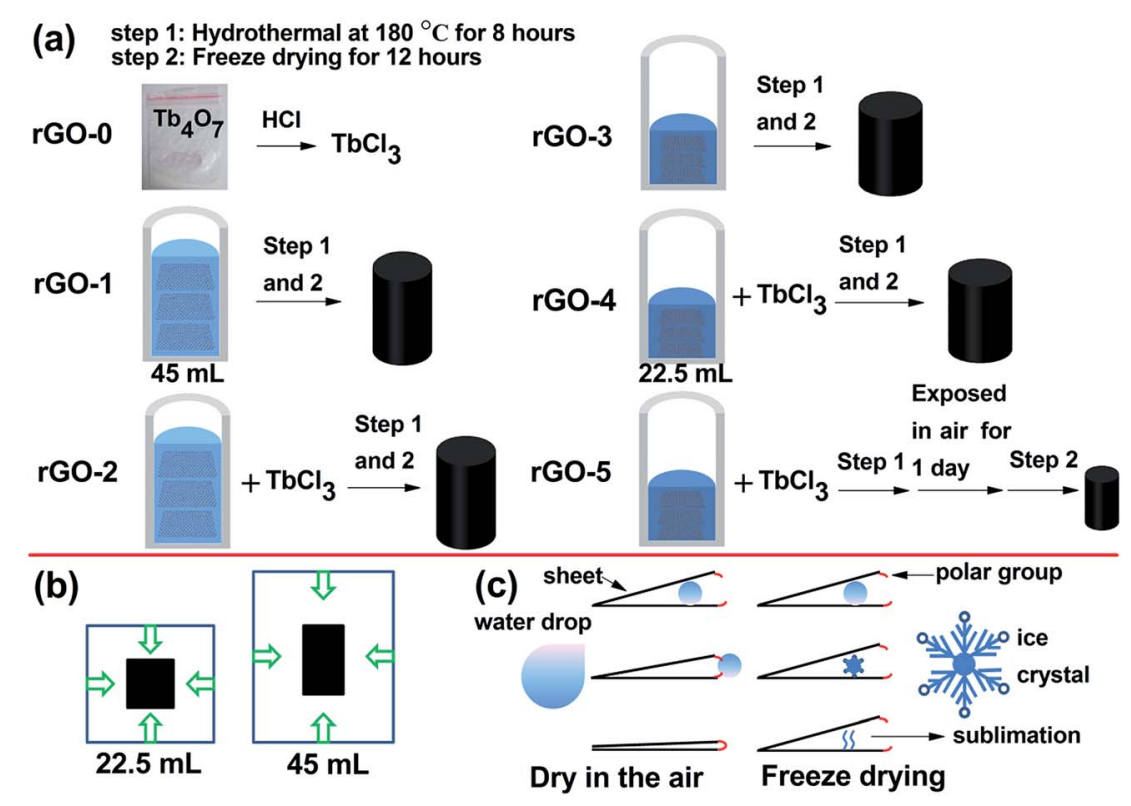

Fig. 1 Drawings of the preparation procedure for the rGO series (a) and schematic diagrams for nucleation (b) and drying methods (c) of rGO. 
Fig. S1c and d. $\dagger$ In these spectra, the XPS signals resulting from Tb 3d $\left(3 \mathrm{~d}_{5 / 2}\right.$ and $\left.3 \mathrm{~d}_{3 / 2}\right)$ orbitals are intricate, including some splitting peaks around the characteristic peaks of the $\mathrm{Tb} 3 \mathrm{~d}$ region, centered at $1242.1 \mathrm{eV}$ and $1275.8 \mathrm{eV}$. The separated doublets for $\mathrm{Tb}^{3+}(1239.1 \mathrm{eV}$ and $1274.9 \mathrm{eV})$ and $\mathrm{Tb}^{4+}(1242.2 \mathrm{eV}$ and $1279.8 \mathrm{eV}$ ) are derived from the hybridization of the $\mathrm{Tb} 4 \mathrm{f}$ orbital with the host orbital and fractional occupancy of the valence $4 \mathrm{f}$ orbital. The relatively intense peaks at $1247.1 \mathrm{eV}$ and $1276.1 \mathrm{eV}$ are assigned to the $3 \mathrm{~d}_{5 / 2}$ and $3 \mathrm{~d}_{3 / 2}$ orbitals of $\mathrm{Tb}^{3+}$, respectively. From the $\mathrm{Tb} 4 \mathrm{~d}$ core level spectrum (Fig. $\mathrm{S} 2 \dagger$ ), three regions are observed for this system, suggesting multiplet splitting in the $4 \mathrm{~d}$ band. The line in the range $148-151 \mathrm{eV}$ is due to $\mathrm{Tb}^{3+}$, those in the range $156-165 \mathrm{eV}$ arise from $\mathrm{Tb}^{4+}$ while the mid part is likely to be related to complex formation between $\mathrm{Tb}, \mathrm{O}$, and C. Finally, it is obvious that Tb has been introduced into the composite and the calculated content of $\mathrm{Tb}$ is about 1\%, as shown in Fig. S1. $\dagger$

A Raman spectrum of the product is shown in Fig. S3a. $\dagger$ The D peak $\left(1345 \mathrm{~cm}^{-1}\right), \mathrm{G}$ peak $\left(1590 \mathrm{~cm}^{-1}\right)$ and 2D peak $\left(2690 \mathrm{~cm}^{-1}\right)$, used to describe the quality of graphene, indicate that the as-prepared material is stacked by graphene sheets. Fig. S3c $\uparrow$ shows the XRD spectrum of rGO-2; the diffraction peak appearing at about 20 degrees originates from the lattice plane (002) while the position of GO is at 10 degrees. This result supports the fact that GO has been changed into rGO (the interplanar spacing decreases from 8-9 $\mathrm{nm}$ to $4-5 \mathrm{~nm}$ ). Some typical molecular vibration patterns of the material are observed in the FTIR spectra (Fig. S3a $\uparrow$ ). Going from high to low wavenumber, there is a region at $3438 \mathrm{~cm}^{-1}$, attributed to the O-H stretching vibration, the weak $1713 \mathrm{~cm}^{-1}$ peak corresponding to $\mathrm{HO}-\mathrm{C}=\mathrm{O}$, and the $1560 \mathrm{~cm}^{-1}$ peak due to unoxidized $\mathrm{sp}^{2} \mathrm{C}=\mathrm{C}$, as well as the $1155 \mathrm{~cm}^{-1} \mathrm{C}-\mathrm{O}$ vibration peak. The TEM image of rGO-2 is shown in Fig. S3d. $\dagger$ It can be seen that the composite is stacked by sheets and that the thickness is about $5.1 \mathrm{~nm}$ (Fig. S7†) on the margin, namely that it is thick in the middle and thin in both sides. All results suggest that the composite is mainly constituted of rGO and that Tb ions have already been doped in the composite.

Commonly, the microstructure is related to the volume. We obtained rGO-3 and rGO-4 by water regulation (reducing the volume of water), as shown in Fig. 1a. It is important to note that the dehydration and skeleton collapse happen in the air for these hydrogels with elapsing time; rGO-5 is obtained due to this effect (Fig. $\mathrm{S} 4 \dagger$ ). Table 1 shows the monolith volumes and BET data for rGO-2, rGO-4 and rGO-5. Surprisingly, the mesoporous volumes and monolith volumes do not match. As Fig. 1b depicts, the monolith volume is inclined to relate to the nucleation area while the mesoporous volume is related to dehydration progress. To better understand the reduction of surface area by dehydration, we studied the pore structure and morphology of rGO-2. The $\mathrm{N}_{2}$-sorption isotherm of rGO-2 is fitted in Fig. 2c, and is categorized as Type 3 according to IUPAC classifications of mesoporosity (from $2 \mathrm{~nm}$ up to $50 \mathrm{~nm}$ ) and macroporosity (exceeding $50 \mathrm{~nm}$ ). This result agrees with the size distribution, as shown in Fig. 2d, suggesting that mesoporosity is formed by stacking. In Fig. $2 a$ and b, the material displays randomly distributed thick sheet-like petals at the micro level, forming a coralline structure with many channels. After magnifying the picture 20 times, finer and dense vortexlike macropores can be observed and the sheets seem thinner at the edge of the extension, which produces greater interlayer spacing. Due to having a smaller nucleation area, more sheets of rGO-4 take part in stacking compared with those in rGO-2, resulting in an enhancement in surface area. As shown in Fig. 1c, in the atmospheric evaporation period, the sheets of rGO-5 are pulled together by their affinity for the droplet, leading to the annihilation of mesoporosity and collapse of the skeleton. However, the shape and the structure of rGO-4 change imperceptibly in the freeze drying, due to the phenomenon of ice's rapid sublimation.

The vibrating sample magnetometer was used to investigate the magnetism of the composite, as shown in Fig. 3. It is obvious that there is paramagnetism in rGO-0 due to its Curie point being below room temperature. There are two states in rGO-1: the weak paramagnetism at the beginning stage and the diamagnetism during the later magnetic field stage, which is attributed to the integrated contribution from the net magnetic moment and diamagnetic moment. Meanwhile the diamagnetic signal faded away when $\mathrm{Tb}$ ions were added, as shown in rGO-2. Compared with rGO-0, rGO-1 and rGO-2 both have a hysteresis loop, indicating that the hysteresis originates from rGO. To study the intrinsic magnetism of rGO and the role of $\mathrm{Tb}$ ions in the composite, we measured the magnetism of rGO-3, rGO-4 and rGO-5 and calculated the magnetic susceptibility $(\chi)$, as shown in Table 1 . It was found that rGO- 1 and rGO-3 have the same shape except for $\chi$, as do rGO- 2 and rGO- 4 , while there is no loop in rGO-5. To understand the phenomena, we refer to the well-known organic polymer model of a quasi-onedimensional organic polymer, in which it is believed that the magnetism originates from the strong coupling between the radical and the $\mathrm{C}=\mathrm{C}$ bond. ${ }^{17}$ Combined with the BET data, it was found that the porosity and the $\mathrm{sp}^{2}$ structure are critical for the loops in the bulk material. This may be explained by stacking from the van der Waals bond in the $z$ direction, destroying the coupling with unpaired electrons. It was also found that paramagnetism appears in all the composites doped

Table 1 The geometric parameters and magnetic susceptibilities of rGO 0-5

\begin{tabular}{llllll}
\hline & rGO-0 & rGO-1 & rGO-2 & rGO-3 & rGO-4 \\
\hline${\text { Monolith volume }{ }^{a}\left(10^{-6} \mathrm{~m}^{3}\right)}_{\text {Specific surface area }\left(\mathrm{m}^{2} \mathrm{~g}^{-1}\right)}$ & & 3.18 & 2.83 & 2.45 & 2.26 \\
$\chi^{a}\left(10^{-7}\right)$ & 732 & -4.54 & 25.3 & & 0.9 \\
\end{tabular}

${ }^{a}$ The magnetic susceptibility at the end of the loop. 
(a)

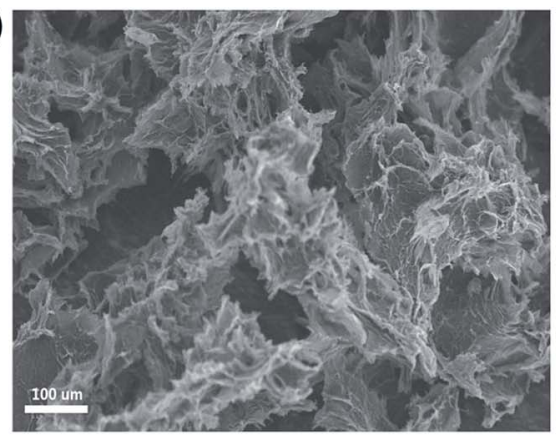

(c)

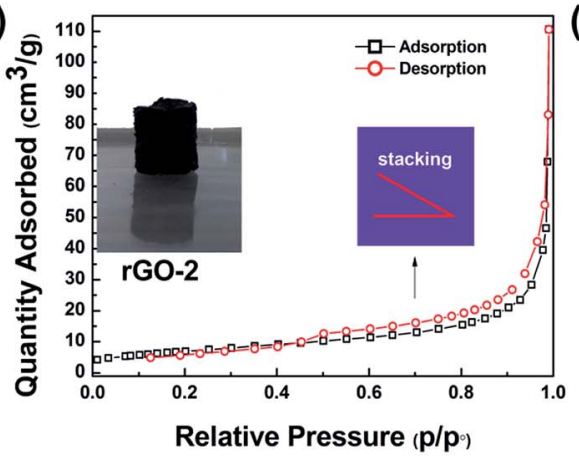

(b)

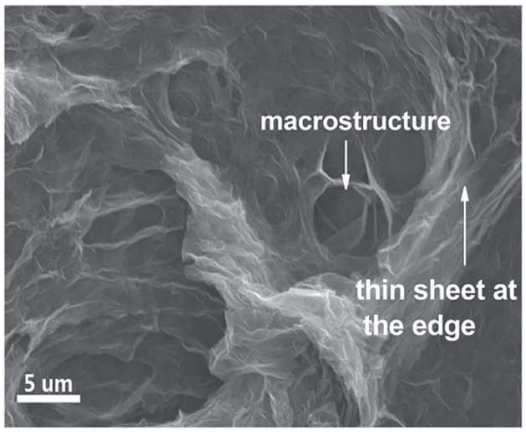

(d)

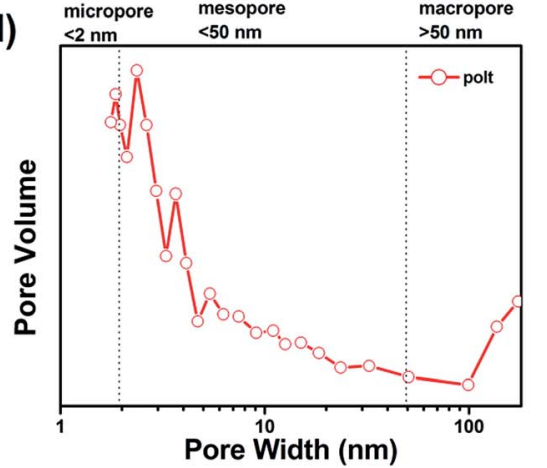

Fig. 2 The different resolution SEM images at (a) $100 \mu \mathrm{m}$ and (b) $5 \mu \mathrm{m}$, BET spectrum (c) and the size distribution (d) of rGO-2.

with $\mathrm{Tb}$ ions, suggesting the regulating effect of the $\mathrm{Tb}$ ions in magnetism. So we successfully integrated paramagnetism into rGO.
Another enhancement for rGO-2 is the luminescence compared with that of rGO-1. There is no light emission for rGO-1 in the visible range. In contrast, rGO-2 has excellent
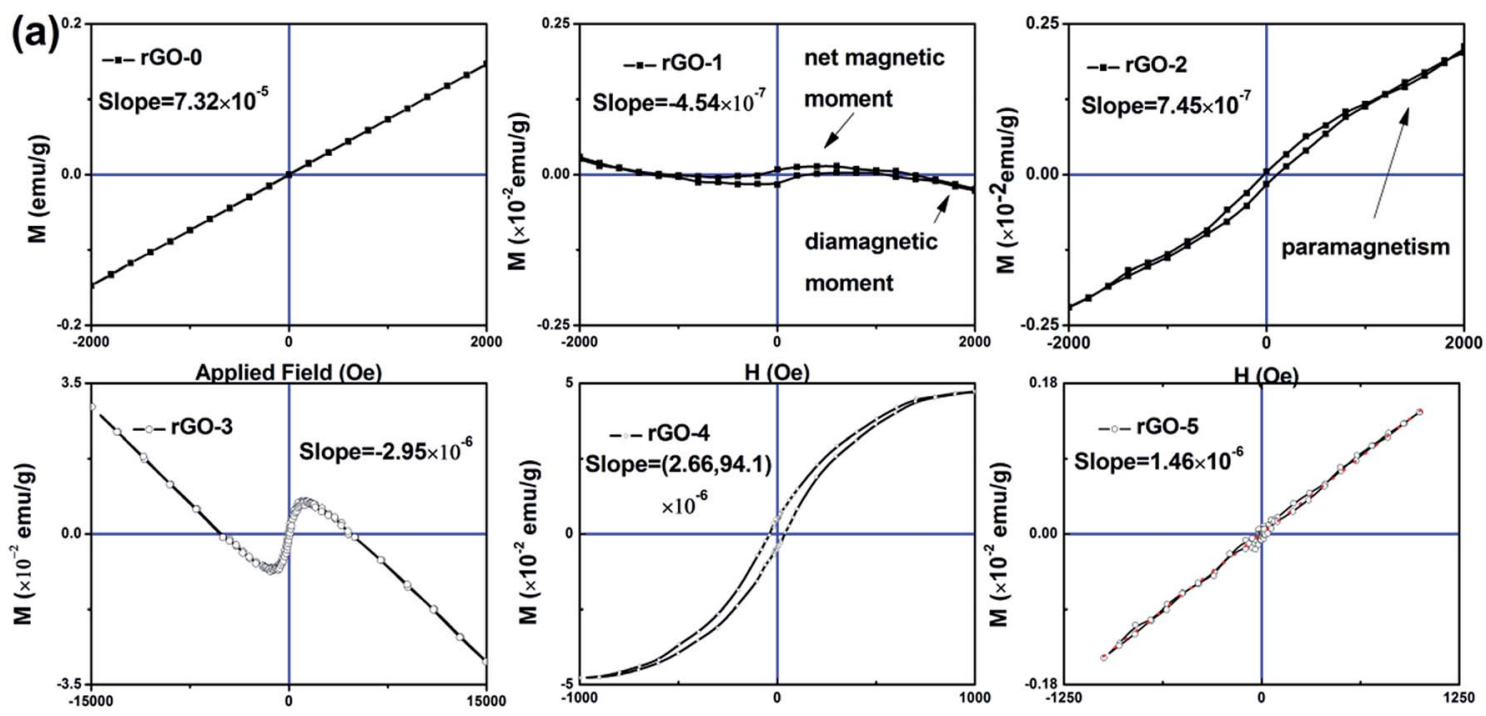

(b)
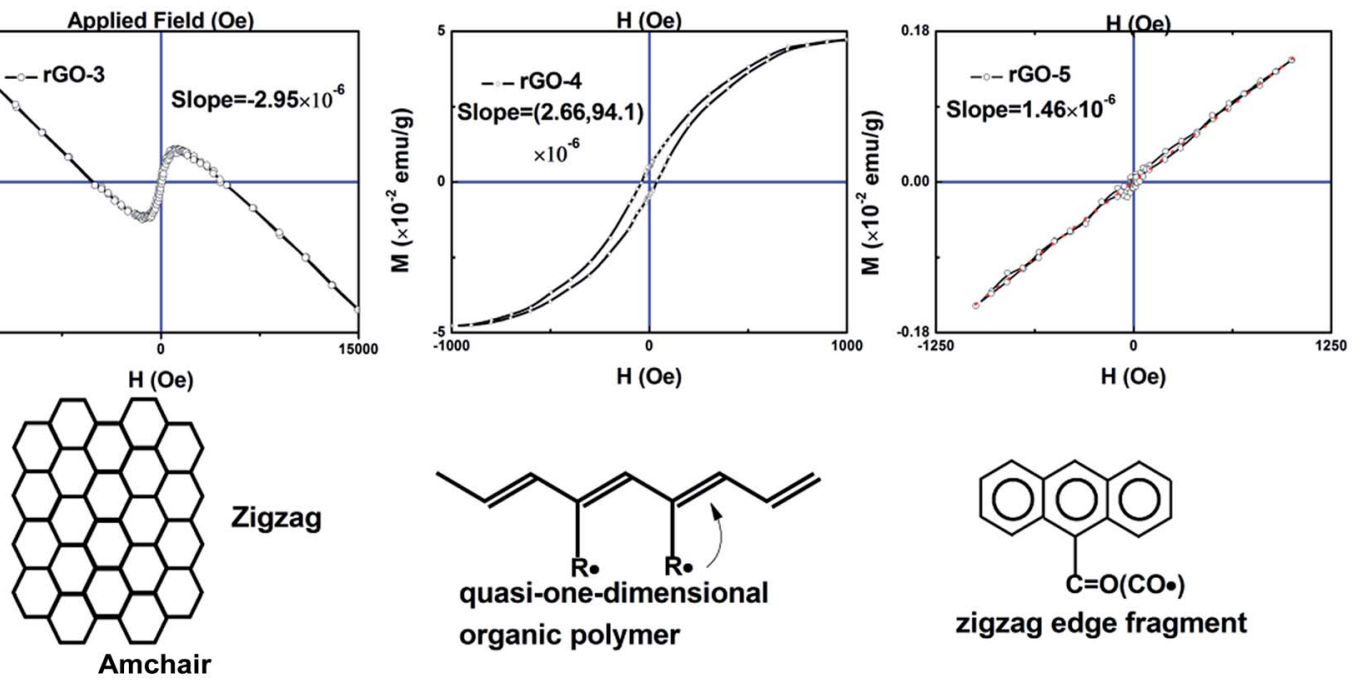

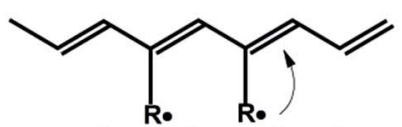

quasi-one-dimensional organic polymer

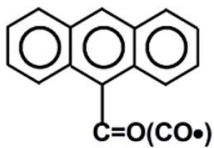

zigzag edge fragment

Fig. 3 The hysteresis loops of the rGO series (a) and the possible models of rGO with magnetism (b). 

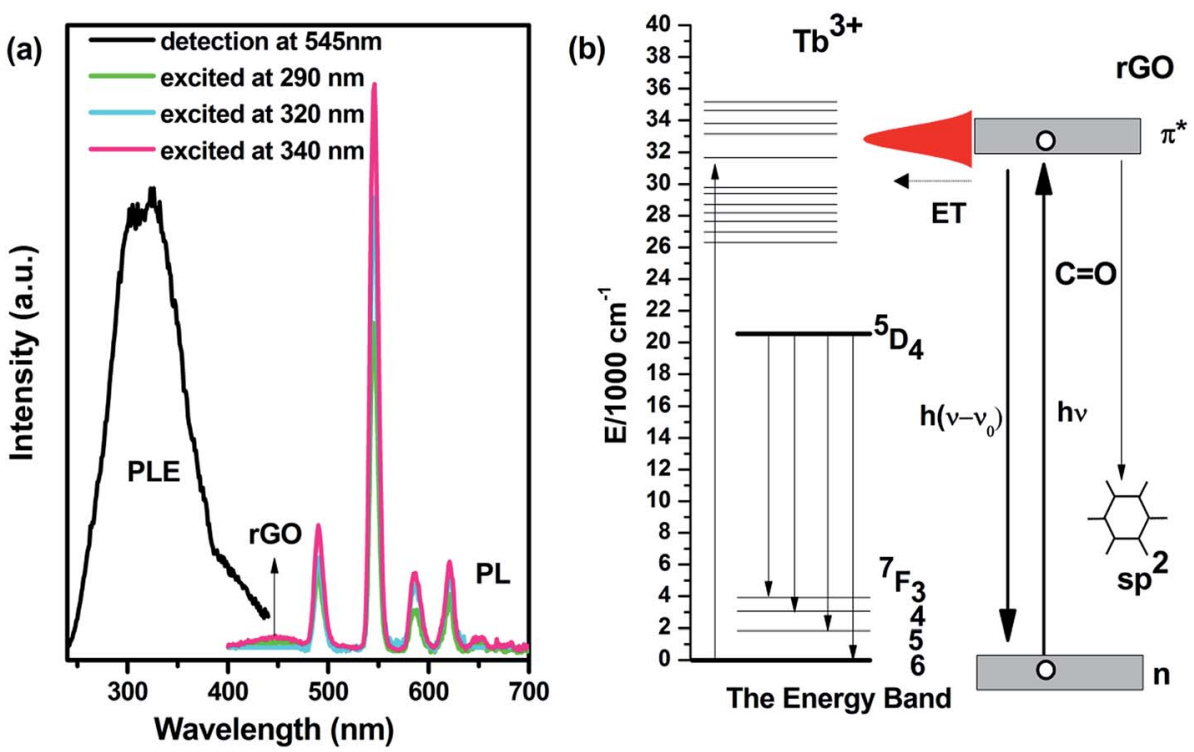

Fig. 4 (a) Photoluminescence excitation and emission spectra of $\mathrm{rGO}-2$. (b) The diagrammatic drawing of the possible transitions during photoluminescence of $\mathrm{rGO}-2$.

photoluminescence (PL) and wide photoluminescence excitation (PLE), as shown in Fig. 4a. It is obvious that rGO-2 has narrow emissions centered at 489, 544, 585 and $621 \mathrm{~nm}$, as well as the weak wide band ranging from $400-480 \mathrm{~nm}$, leading to intense green luminescence with weak blue light. These originated from the ${ }^{5} \mathrm{D}_{4}{ }^{-} \mathrm{F}_{J}(J=3,4,5,6)$ transitions of $\mathrm{Tb}$ ions and the $\mathrm{sp}^{2}$ structure of rGO, respectively. ${ }^{18,19}$ And the PLE spectra (monitored at $544 \mathrm{~nm}$, detailed in Fig. S5 $\dagger$ ) show a broad excitation region ranging from $250-400 \mathrm{~nm}$, which is different from rGO-0 which is mainly excited in discrete peaks..$^{16}$ The band gap of graphene measured by UV-vis absorption spectroscopy was used to confirm the variation in PLE. In Fig. S6, $\uparrow$ a widening peak appears at about $300 \mathrm{~nm}$ from $\mathrm{rGO}-1$, which is assigned to the $\mathrm{n}-\pi^{*}$ transition of $\mathrm{C}=\mathrm{O}$. At the same time, $\mathrm{Tb}$ ions tend to bond with the oxygen functional group. As Fig. $4 \mathrm{~b}$ shows, there is probably energy transfer between $\mathrm{Tb}$ ions and rGO-2. Meanwhile, another luminescence center of rGO should be ascribed to the contribution of the local $\mathrm{sp}^{2}$ structure, which is more like carbon dots. These results suggest that the additional functionality has been endowed to our composite and $\mathrm{Tb}$ ions locate near to the $\mathrm{C}=\mathrm{O}$ group.

\section{Conclusion}

In summary, a material, reduced graphene oxide doped with $\mathrm{Tb}$ ions, was synthesized simply, and it is three dimensional, has a mesoporous structure, and possesses paramagnetism and photoluminescence properties. By introducing $\mathrm{Tb}$ ions into $\mathrm{rGO}$, the material was endowed with paramagnetism and photoluminescence properties, suggesting its potential application in controllable synthesis and optical detection fields. Furthermore, we demonstrated energy transfer between $\mathrm{Tb}$ ions and $\mathrm{rGO}$, as well as locating the binding site of $\mathrm{Tb}$ ions in $\mathrm{rGO}$, which provides the potential to extend to other rare earth compounds.

\section{Conflicts of interest}

There are no conflicts to declare.

\section{Acknowledgements}

This work was financially supported by the National Science Foundation of China (51402140, U1632129, and 51602137) and the Fundamental Research Funds for the Central Universities (lzujbky-2016-128).

\section{References}

1 F. Guo, M. Creighton, Y. Chen, R. Hurt and I. Külaots, Carbon, 2014, 66, 476-484.

2 F. Sharif, L. R. Gagnon, S. Mulmi and E. P. L. Roberts, Water Res., 2017, 114, 237-245.

3 M. Yang, N. Zhao, Y. Cui, W. Gao, Q. Zhao, C. Gao, H. Bai and T. Xie, ACS Nano, 2017, 11, 6817-6824.

4 W. Gao, N. Zhao, W. Yao, Z. Xu, H. Bai and C. Gao, RSC Adv., 2017, 7, 33600-33605.

5 X. Zhao, W. Yao, W. Gao, H. Chen and C. Gao, Adv. Mater., 2017, 29, 1701482.

6 W. Chen, S. Li, C. Chen and L. Yan, Adv. Mater., 2011, 23, 5679-5683.

7 Z. Niu, J. Chen, H. H. Hng, J. Ma and X. Chen, Adv. Mater., 2012, 24, 4144-4150.

8 R. Sitko, E. Turek, B. Zawisza, E. Malicka, E. Talik, J. Heimann, A. Gagor, B. Feist and R. Wrzalik, Dalton Trans., 2013, 42, 5682-5689.

9 J. Liu, X. Ge, X. Ye, G. Wang, H. Zhang, H. Zhou, Y. Zhang and H. Zhao, J. Mater. Chem. A, 2016, 4, 1970-1979.

10 G. Singh, A. Choudhary, D. Haranath, A. G. Joshi, N. Singh, S. Singh and R. Pasricha, Carbon, 2012, 50, 385-394. 
11 J. Su, M. Cao, L. Ren and C. Hu, J. Phys. Chem. C, 2011, 115, 14469-14477.

12 W. Han, R. K. Kawakami, M. Gmitra and J. Fabian, Nat. Nanotechnol., 2014, 9, 794.

13 Y.-J. Li, M. Wang, M.-y. Tang, X. Tian, S. Gao, Z. He, Y. Li and T.-G. Zhou, Phys. E, 2016, 75, 169-173.

14 J. Li, X. Li, P. Zhao, D. Y. Lei, W. Li, J. Bai, Z. Ren and X. Xu, Carbon, 2015, 84, 460-468.

15 D. Wang, H. Gao, E. Roze, K. Qu, W. Liu, Y. Shao, S. Xin and Y. Wang, J. Mater. Chem. C, 2013, 1, 5772-5778.
16 K. Chen, H. Gao, B. Bai, W. Liu and X. Li, Nanoscale Res. Lett., 2017, 12, 204.

17 W. Wang, Z. Liu and K. Yao, Phys. Rev. B: Condens. Matter Mater. Phys., 1997, 55, 12989.

18 G. Eda, Y. Y. Lin, C. Mattevi, H. Yamaguchi, H. A. Chen, I. Chen, C. W. Chen and M. Chhowalla, Adv. Mater., 2010, 22, 505-509.

19 H. Gao, Y. Zhou, K. Chen and X. Li, Mater. Res. Bull., 2016, 77, 111-114. 